TECHNICAL NOTE

\title{
Comparative analysis of various fixative solutions on insect preservation for molecular studies
}

\author{
Matteo Bisanti, Sonia Ganassi \& Mauro Mandrioli* \\ Dipartimento di Biologia Animale, Università di Modena e Reggio Emilia, Via Campi 213/D, 41100 Modena, Italy
}

Accepted: 7 October 2008

Key words: DNA analysis, entomological collection, acetone preservation, Coleoptera, Tenebrionidae, Tenebrio molitor, sensilla, morphology

\section{Introduction}

In the past few years, several methods have been elaborated in order to obtain DNA for biomolecular studies from insects belonging to museum collections (Cooper, 1994; Hillis et al., 1996; Vogler \& Pearson, 1996; Carter et al., 1997; Fukatsu, 1999; Quicke et al., 1999; Dean \& Ballard, 2001). The quality and quantity of DNA extracted from museum specimens are extremely variable. One particular common problem is the occurrence of DNA shearing; this results in the degradation of DNA into small fragments, which are responsible not only for poor quality of the extracted DNA, but also for failure in subsequent polymerase chain reaction (PCRs) (Dessauer et al., 1990; Lindahl, 1993; Golenberg et al., 1996; Hofreiter et al., 2001). DNA preservation is affected by various parameters including environmental temperature, $\mathrm{pH}$, exposure to ultraviolet (UV), and age of the samples (Dessauer et al., 1990; Lindahl, 1993). Therefore, insects that are not immersed in fixative solutions may be extremely fragile, and are frequently useless for molecular analyses.

Because DNA extraction procedures are usually destructive, it is important to find a balance between the maintenance of irreplaceable specimens and the 'added value' that molecular analysis may bring to the studied samples (Payne \& Sorenson, 2003; Mandrioli, 2008). The availability of inexpensive and suitable methods for preserving insect samples is essential, considering that recently DNA barcoding projects have started in several countries, yielding large numbers of insect specimens that need to be stored (Hebert et al., 2002; Savolainen et al., 2005).

At present, ultra-cold freezing at $-80{ }^{\circ} \mathrm{C}$ and liquid nitrogen are possibly the two most effective methods for

*Correspondence: Matteo Bisanti, Dipartimento di Biologia Animale, Università di Modena e Reggio Emilia, Via Campi 213/D, 41100 Modena, Italy. E-mail: mandrioli.mauro@unimo.it specimen storage and maintenance, as both give results in terms of DNA extraction and PCR amplification that are very similar to those obtained using fresh samples (Lindahl, 1993; Dillon et al., 1996; Quicke et al., 1999; Mandrioli et al., 2006). These methods, however, are not always available and it may be difficult to apply them during field surveys or when storing a large number of samples. Both methods also require constantly controlled storage conditions, which can be time-consuming and costly.

Taking the above into account, several alternative and more practical methods have been examined to preserve insect samples for DNA studies (Post et al., 1993; Dessauer et al., 1990; Fukatsu, 1999; Dean \& Ballard, 2001). There are, however, some discrepancies in the published results, and a comparative and exhaustive analysis of the effects of various fixative solutions on insect preservation for molecular analyses has been performed in only a few taxa (Dillon et al., 1996; Quicke et al., 1999; Mandrioli et al., 2006). For example, cold $70 \%$ and $100 \%$ ethanol solutions are reported to be highly effective in some hymenopteran species (Quicke et al., 1999), whereas both were only rarely effective in Lepidoptera (Mandrioli et al., 2006) or Coleoptera (Reiss et al., 1995), suggesting that the best fixative solutions for insects may differ depending on cuticle composition (Mandrioli, 2008).

In order to establish a convenient and reliable method for DNA preservation that can be applied both during field collection and storage of collected insects, we carried out a comparative analysis of fixative solutions, verifying both the quality and quantity of DNA extracted from insects stored for different periods of time. Furthermore, we verified whether fixative solutions affected PCR amplification performed with the extracted DNA samples. Finally, we studied the influence of the fixative solution on preservation of morphological characters, in particular the fragile sensilla. As experimental model, we used the yellow mealworm beetle, Tenebrio molitor L. (Coleoptera: 
Tenebrionidae), comparing the results obtained with this beetle species to those available in the literature for other insects.

\section{Materials and methods}

\section{Material and preservation conditions}

Tenebrio molitor adults, collected from a laboratory colony in Modena (Italy), were stored at room temperature after immersion in various standard-grade fixative solutions (acetone, Carnoy's, and 75\% and 100\% ethanol) or stored in an ultracold freezer and liquid nitrogen. Specimens immersed in preservative solutions were placed immediately after capture in a plastic 2-ml cryovial, tightly closed with a plastic lid, and stored for time periods ranging from 1 day to 18 months before DNA extraction and PCR amplification. Each vial was wrapped with chloroformresistant parafilm in order to avoid evaporation of the fixative. Fixative levels in the vials were checked monthly in order to ensure that specimens remained wholly immersed in the fixative and new fixative was added if necessary. Fresh beetles were used as controls and extracted immediately after capture, whereas a set of T. molitor specimens was stored as dried insects at room temperature within a standard entomological box, to compare the results obtained with these insects to those obtained with beetles preserved in fixative solutions. Ten specimens were stored for each preservation method for each period of preservation $(1,2$, and 4 days, 1 and 2 weeks, and $1,2,4,6,12$, and 18 months). The effects of the various fixative solutions were evaluated using three parameters: quality of the extracted DNA, DNA quantity, and success of coxI PCR amplification.

\section{Extraction and electrophoretic analysis of genomic DNA}

Genomic DNA was isolated from whole adults of equal size according to Mandrioli (2002). Specifically, beetles were homogenized in tetrasodium 2-[2-(bis(carboxylatomethyl) amino)ethyl-(carboxylatomethyl)amino]acetate (S-EDTA) buffer ( $0.1 \mathrm{~m} \mathrm{NaCl}, 50 \mathrm{~mm}$ EDTA, $\mathrm{pH}$ 8.0). Sodium dodecyl sulphate $1 \%$ and $100 \mu \mathrm{g} / \mathrm{ml}$ proteinase $\mathrm{K}$ solutions were added to the homogenate and the mixture was incubated at $55^{\circ} \mathrm{C}$ overnight. One volume of phenol-chloroformisoamylic alcohol (25:24:1) was added and the mixture was gently stirred and then centrifuged $(12000 \mathrm{~g})$ for $5 \mathrm{~min}$. The supernatant was treated twice as described above, digested with RNase $(100 \mu \mathrm{g} / \mathrm{ml})$ for $30 \mathrm{~min}$ at $37^{\circ} \mathrm{C}$, ethanol precipitated, washed three times with $70 \%$ ethanol, air dried, and re-suspended in sterile bi-distilled water. Electrophoretic runs were carried out at $60 \mathrm{~V}$ for $2 \mathrm{~h}$ on $1.2 \%$ (wt/vol) agarose gels in standard Tris-borate-EDTA buffer, stained with ethidium bromide, and observed on
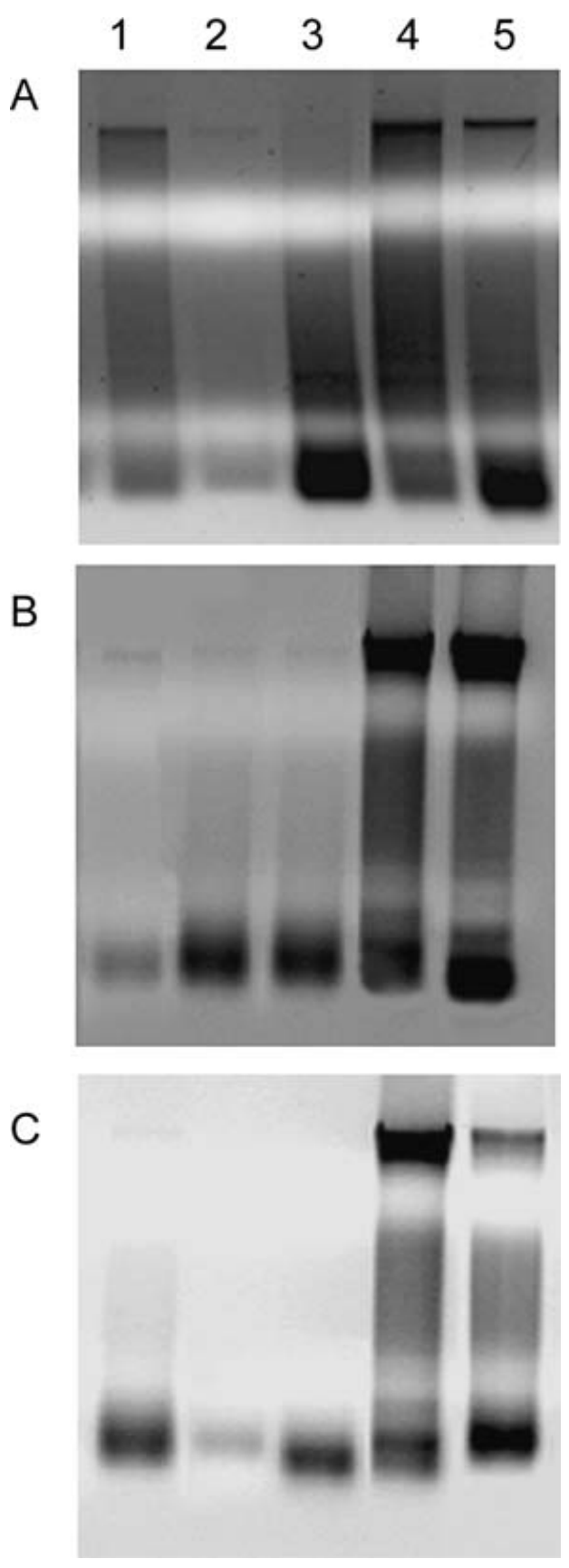

Figure 1 Electrophoresis of DNA samples extracted from specimens preserved as (lane 1) dried and immersed in (2) Carnoy solution, (3) 75\% ethanol, (4) acetone, and (5) absolute ethanol after (A) 1 day, (B) 2 weeks, and (C) 18 months.

a UV transilluminator (Biorad, Woodinville, WA, USA). DNA quantification was performed using a nanodrop spectro-photometer (Thermo Fisher scientific, Waltham, MA, USA) and data were analyzed using Microsoft Office Excel software (Microscoft Corporation, Washington, USA). 


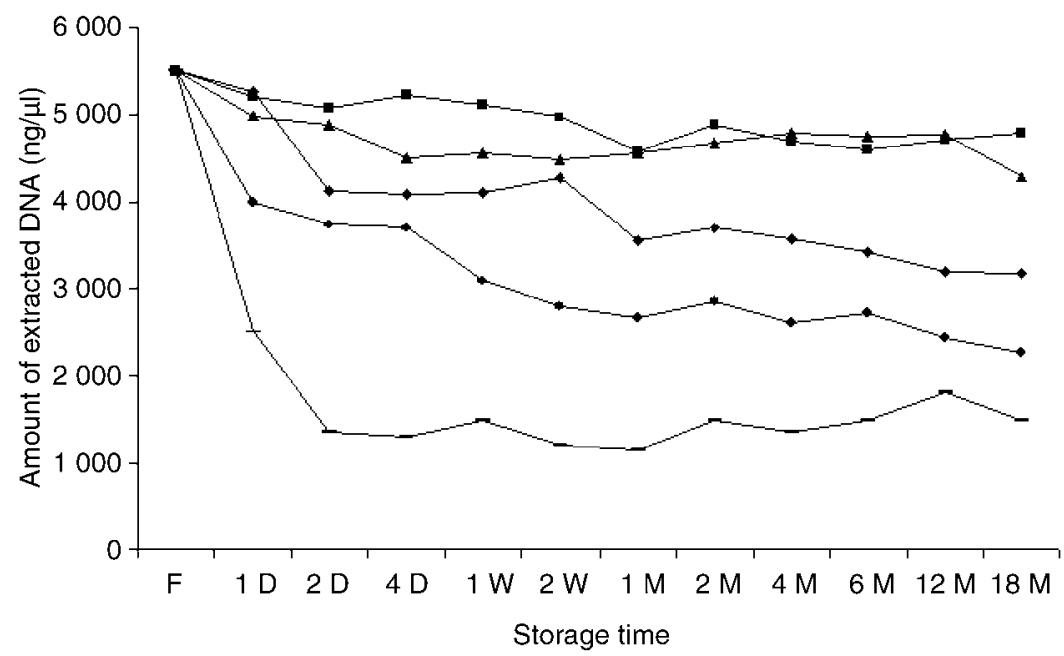

Figure 2 Graphical representation of DNA preservation for each fixative solution at different storage times. F, fresh sample; D, day; W, week; M, month. Studied fixatives: acetone $(\boldsymbol{\square})$, absolute ethanol $(\boldsymbol{\Lambda})$, $75 \%$ ethanol (•), Carnoy solution $(\bullet)$, and dried specimens (-).

\section{Polymerase chain reaction amplification}

A portion of the coxI gene was amplified for each extracted DNA sample, using two universal primers for coleopteran coxI gene: primer Pat (5'-TCCAATGCACTAATCTGCCATA$\left.3^{\prime}\right)$ and Jerry (5'-CAACATTTATTTTGATTTTTTG-3') (Simon et al., 1994). Amplifications were performed with a Hybaid thermal-cycler (Waltham, MA, USA) at annealing parameters corresponding to $45^{\circ} \mathrm{C}$ for $90 \mathrm{~s}$, whereas the denaturation was performed at $94{ }^{\circ} \mathrm{C}$ for $60 \mathrm{~s}$ and amplification at $72{ }^{\circ} \mathrm{C}$ for $60 \mathrm{~s}$. The amplification mix contained $100 \mathrm{ng}$ of genomic DNA, $1 \mu \mathrm{M}$ of each primer, $200 \mu \mathrm{M}$ dNTPs, and $2 \mathrm{U}$ of DyNAZyme II polymerase (Finnzymes Oy, Espoo, Finland). PCR products were electrophoresed at $70 \mathrm{~V}$ for $90 \mathrm{~min}$ on $1.2 \%$ agarose gels in standard Tris Acetic Acid EDTA buffer, stained with ethidium bromide, and observed on a UV transilluminator according to Mandrioli et al. (2006).

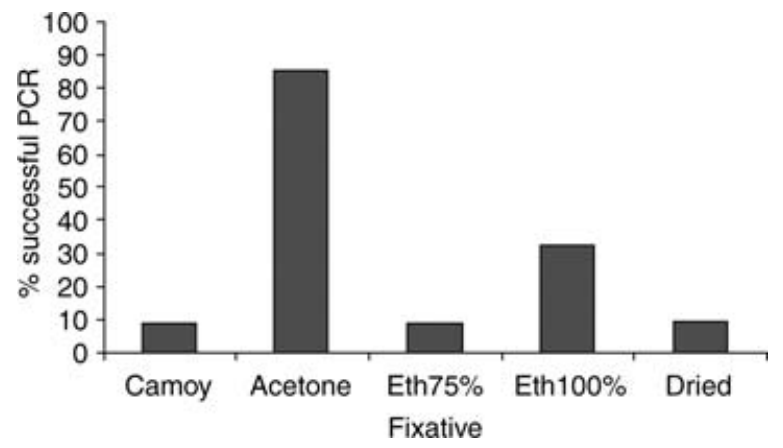

Figure 3 Percentage of successful polymerase chain reaction (PCR) for each fixative solution after PCR, representing the share of samples that yielded coxI amplification for each fixative.

\section{Scanning electron microscopy analysis of preserved insects}

The samples of T. molitor, maintained in pure acetone for 18 months, were washed with pure acetone before observation, until the acetone was completely clean. During washes, the samples were gently agitated on a stirrer. The samples were put on filter paper to draw off surplus acetone and then maintained under an inhaling cap until the acetone evaporated completely. Samples were then mounted on aluminium stubs and sputter-coated with $10 \mathrm{~nm}$ gold-palladium in a Coating Unit E 500 (Polaron, Cambridge, UK) and observed under a Philips XL-30 scanning electron microscope (Philips Electronic Instruments Company, Mahwah, NJ, USA).

\section{Results and discussion}

As reported in previous articles (Dessauer et al., 1990; Post et al., 1993; Fukatsu, 1999; Dean \& Ballard, 2001; Mandrioli et al., 2006), Diptera, Hymenoptera, and Hemiptera preserved in $75 \%$ ethanol and Carnoy solutions resulted in highly fragmented DNA in each sample, including the 1-day-old specimens; this indicated that DNA shearing started immediately after insect death and that both these solutions failed to preserve DNA. Similar results were obtained with the lepidopteran Mamestra brassicae (L.) (Mandrioli et al., 2006), indicating that both Carnoy solution and 75\% ethanol probably enter the insect tissues too slowly, thus affecting DNA preservation. This result is important, because $75 \%$ ethanol is frequently used as a fixative for insect archival maintenance in museum collections or laboratories, whereas it actually does not work properly. The amount of DNA extracted from insects preserved in 75\% ethanol was reduced by about $20 \%$ in the first 2 days and by $50 \%$ after 

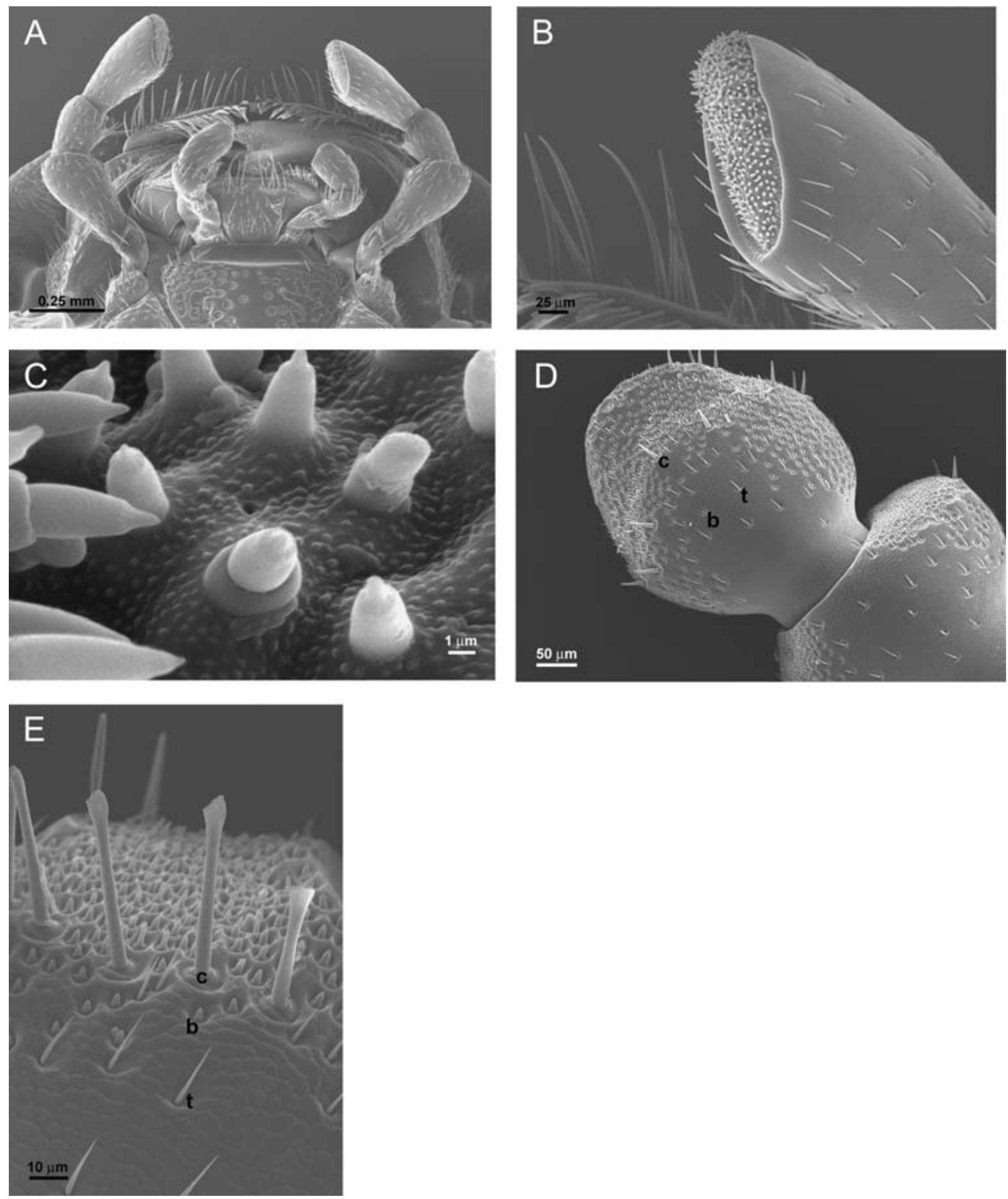

Figure 4 Scanning electron microscope micrographs of Tenebrio molitor, 18-month-old specimens preserved in acetone. (A) Ventral view of the mouthparts. (B) Distal segment of the maxillary palp with sensilla basiconica. (C) Sensilla basiconica of the distal segment of the maxillary palp. (D) Dorsal surface of antennal segment 11 of a male. (E) Sensilla on dorsal surface of antennal segment 9. Sensilla basiconica (b), sensilla trichodea (t), and sensilla chaetica $(c)$. 

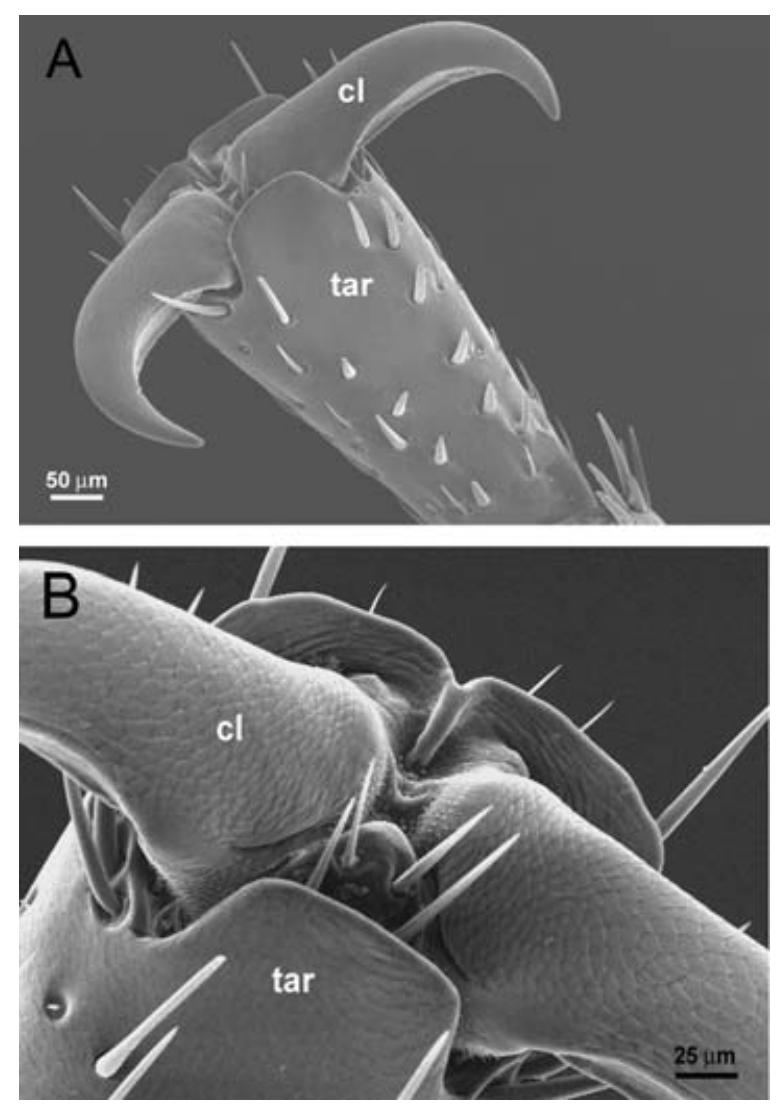

Figure 5 Scanning electron microscope micrographs of Tenebrio molitor, 18-month-old specimens preserved in acetone. (A) Ventral view of tarsal segment (tar) and pretarsus with claws (cl). (B) Pretarsus with claws.

2 weeks (Figures 1 and 2). After 2 weeks, the DNA quantity seemed to stabilize, indicating that DNA shearing had been reduced (Figure 2).

DNA quality was significantly higher if obtained with absolute ethanol or acetone at room temperature, as both preserved a large amount of DNA (comparable to the two-fixative solution). However, acetone seemed to preserve more DNA at high molecular weight than absolute ethanol, indicating that partial DNA degradation still occurred in specimens preserved in absolute ethanol. Acetone can quickly permeate insect tissues, leading to fast and effective DNA preservation so that the percentage of samples that gave good DNA extraction was higher with acetone than with absolute ethanol.

Even if the results with acetone were better than those with absolute ethanol, ethanol-preserved insects gave a great deal of high-quality DNA for molecular studies, also after 18 months. This is in contrast to the data published by Reiss and colleagues, who reported that various beetle species cannot be stored in ethanol for more than 6 weeks for DNA studies (Reiss et al., 1994) (Figure 2). Our results are in agreement with those describing the successful extraction of hymenopteran DNA from whole specimens stored in ethanol for several years (Quicke et al., 1999), suggesting a possible taxon-specific effect of ethanol penetration in the specimens.

Acetone gave positive results, assuring both good quality and quantity of the extracted DNA samples. Our results reinforced those obtained by Fukatsu (1999) in aphids and by Mandrioli et al. (2006) in moths, indicating that acetone may assure quick and effective insect preservation.

Storage of dried specimens at room temperature quickly led to shearing of DNA, reducing the amount to $50 \%$ after 1 day and to $20 \%$ after 2 days (Figure 2). Nevertheless, after 2 days the amount of DNA remained constant until 18 months of preservation, suggesting that complete desiccation was attained after 2 days and that only after 2 days DNA was fixed sufficiently. In museum collections, most Coleoptera are kept as air-dried specimens. Our results indicated that stored desiccated specimens cannot be used for molecular studies, as extensive DNA degradation precedes desiccation of the intact insect.

Recently, Gilbert et al. (2007) reported that even after 40 years of storage of dried Coleoptera at room temperature, it was possible to extract DNA useful for PCR. In order to verify whether the fixative choice can affect the possibility of using museum-collected insects for molecular studies, such as barcoding projects, we performed PCR experiments using the coxI. After PCR, dried insects gave successful amplification in several cases (about 10\%) and similar percentages of success have been obtained using DNA extracted from 75\% ethanol- and Carnoy-preserved insects (Figure 3). Thus, dried insects can be used for molecular essays, but it is impossible to know beforehand whether molecular studies will be successful, so that museum curators cannot be sure that sample sacrifice will lead to useful molecular data. Balancing the damage done to the collection and the added value of biomolecular studies, the use of dried samples can be risky, also considering the amount of time, effort, and money that molecular experiments require (Mandrioli, 2008). Higher success rates were obtained with acetone and absolute ethanol; acetonepreserved samples allowed successful PCR amplification of coxI in more than $80 \%$ of the analyzed samples (Figure 3 ).

Previous reports indicated that storage time appears to be the primary factor responsible for DNA degradation not only with dried specimens, but also with fixed ones (Dean \& Ballard, 2001). However, based on our experience, the preservation of insects in water-containing solutions (such as $75 \%$ ethanol) or in fixatives with slow entrance in the insect bodies (such as Carnoy's solution) may yield very poor results after DNA extraction, which is worse 

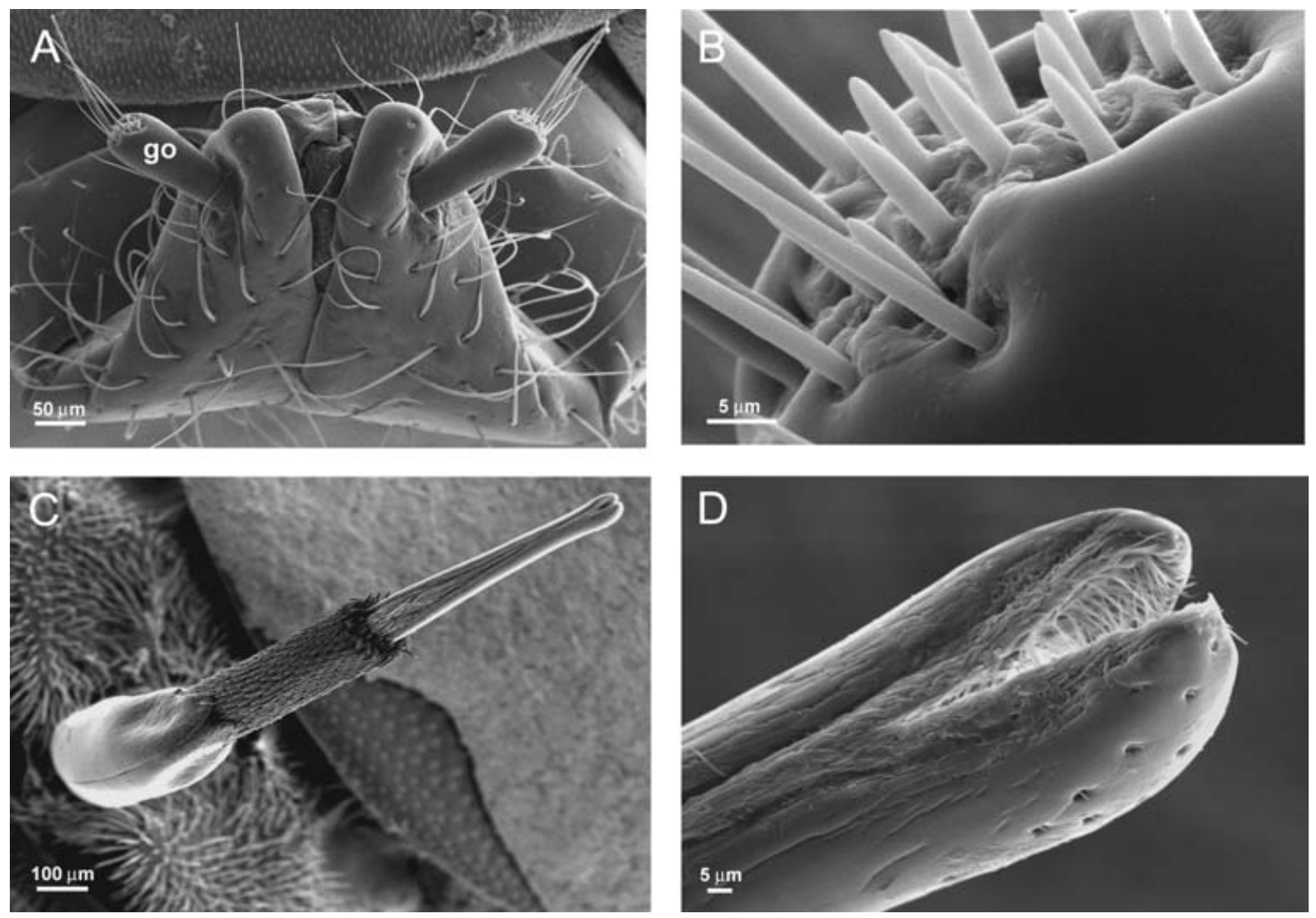

Figure 6 Scanning electron microscope micrographs of Tenebrio molitor, 18-month-old specimens preserved in acetone. Female reproductive system: (A) dorsal view of ovipositor and associated structures (gonostylus, go); (B) detail of the gonostylus with sensilla. Male reproductive system: (C) dorsal view of male copulatory organ; (D) detail of the distal part of the male copulatory organ.

than those obtained using dried, pinned insects housed in cabinets.

Fukatsu (1999) reported that acetone can also preserve morphological characteristics that allow morphological analyses in aphids. In view of this, we analyzed the structure of sensilla on different parts of the T. molitor body; sensilla are generally considered fragile structures, whose morphology can be altered by inadequate storage or fixation. Scanning electron microscope analysis of 18 -month-old specimens preserved in acetone (Figures 4-6) showed that the morphology of the sensilla was well-preserved compared to those of fresh insects. Furthermore, acetone did not affect coloration of the specimens. As a whole, this indicates that fixation in acetone may also allow morphological studies of beetles.

\section{Acknowledgements}

This work has been subsidized by the 'F.A.R.' grant from the University of Modena and Reggio Emilia (Mauro
Mandrioli). We are greatly indebted to Antonio De Cristofaro for his precious suggestions during the analysis of scanning electron microscope images.

\section{References}

Carter DJ, Vogler AP \& Vane-Wright RI (1997) Notes on basic collecting techniques for morphological and molecular studies of Lepidoptera. Metamorphosis 8: 99-106.

Cooper A (1994) DNA from museum specimens. Ancient DNA (ed. by B Herrmann \& S Herrmann), pp. 149-165. Springer, New York, NY, USA.

Dean MD \& Ballard WO (2001) Factors affecting mitochondrial DNA quality from museum preserved Drosophila simulans. Entomologia Experimentalis et Applicata 98: 279-283.

Dessauer HC, Cole CJ \& Hafner MS (1990) Collection and storage of tissue. Molecular Systematics (ed. by DM Hillis \& C Moritz), pp. 29-47. Sinauer, Sunderland, MA, USA.

Dillon N, Austin AD \& Bartowsky E (1996) Comparison of preservation techniques for DNA extraction from hymenopterous insects. Insect Molecular Biology 5: 21-24. 
Fukatsu T (1999) Acetone preservation: a practical technique for molecular analysis. Molecular Ecology 8: 1935-1945.

Gilbert MT, Moore W, Melchior L \& Worobey M (2007) DNA extraction from dry museum beetles without conferring external morphological damage. PLoS ONE 2: e272.

Golenberg EM, Bickel A \& Weihs P (1996) Effect of highly fragmented DNA on PCR. Nucleic Acids Research 24: 5026-5033.

Hebert PDN, Cywinska A, Ball SL \& de Waard JR (2002) Biological identification though DNA barcodes. Proceedings of the Royal Society of London B 270: 313-321.

Hillis DM, Moritz C \& Mable BK (1996) Molecular Systematics, 2nd edn. Sinauer Associates, Sunderland, MA, USA.

Hofreiter M, Jaenicke V, Serre D, Haeseler AA \& Paabo S (2001) DNA sequences from multiple amplifications reveal artefacts induced by cytosine deamination in ancient DNA. Nucleic Acids Research 29: 4793-4799.

Lindahl T (1993) Instability and decay of the primary structure of DNA. Nature 362: 709-715.

Mandrioli M (2002) Cytogenetic characterization of telomeres in the holocentric chromosomes of the lepidopteran Mamestra brassicae. Chromosome Research 9: 279-286.

Mandrioli M (2008) Insect collections and DNA analysis: how to manage collections? Museum Management and Curatorship 23: 193-199.

Mandrioli M, Borsatti F \& Mola L (2006) Factors affecting DNA preservation from museum-collected lepidopteran specimens. Entomologia Experimentalis et Applicata 120: 239-244.
Payne RB \& Sorenson MD (2003) Museum collections as sources of genetic data. Bonner Zoologische Beiträge 51: 97-194.

Post RJ, Flook PK \& Miles AL (1993) Methods for the preservation of insects for DNA studies. Biochemical Systematics and Ecology 27: 85-92.

Quicke DLJ, Belshaw R \& Lopez-Vaamonde C (1999) Preservation of hymenopteran specimens for subsequent molecular and morphological study. Zoologica Scripta 28: 261-267.

Reiss RA, Schwert DP \& Ashworth AC (1994) Field preservation of Coleoptera for molecular genetic analysis. Environmental Entomology 24: 716-719.

Reiss RA, Schwert DP \& Ashworth AC (1995) Field preservation of Coleoptera for molecular genetic analysis. Environmental Entomology 124: 387-396.

Simon C, Frati F, Beckenbach A, Crespi B, Liu H \& Flook P (1994) Evolution, weighting, and phylogenetic utility of mitochondrial gene sequences and a compilation of conserved polymerase chain reaction primers. Annals of the Entomological Society of America 87: 651-701.

Savolainen V, Cowan RS, Vogler AP, Roderick GK \& Lane R (2005) Towards writing the encyclopaedia of life: an introduction to DNA barcoding. Philosophical Transactions of the Royal Society B 360: 1805-1811.

Vogler AP \& Pearson DL (1996) A molecular phylogeny of the tiger beetles (Cicindelidae): congruence of mitochondrial and nuclear rDNA data sets. Molecular Phylogenetics and Evolution 6: 321-338. 